\title{
Single breath-hold whole heart coronary MRA with isotropic spatial resolution using highly-accelerated parallel imaging with a 32-element coil array
}

\author{
Jian $\mathrm{Xu}^{* 1}$, Daniel Kim², Ricardo Otazo ${ }^{2}$, Benjamin $\mathrm{Ge}^{3}$, Sven Zuehlsdorff4, \\ Xiaoming $\mathrm{Bi}^{4}$, Bernd Stoeckel ${ }^{5}$ and Daniel Sodickson ${ }^{2}$
}

\begin{abstract}
Address: ${ }^{1}$ Siemens Medical Solutions USA Inc. and PolyTechnic Institute of NYU, New York, NY, USA, ${ }^{2}$ Radiology, New York University, NY, USA, ${ }^{3}$ Medical School, New York University, NY, USA, ${ }^{4}$ Siemens Medical Solutions USA Inc., Chicago, IL, USA and ${ }^{5}$ Siemens Medical Solutions USA Inc., New York, NY, USA

* Corresponding author
\end{abstract}

from 13th Annual SCMR Scientific Sessions

Phoenix, AZ, USA. 21 -24 January 2010

Published: 21 January 2010

Journal of Cardiovascular Magnetic Resonance 2010, I2(Suppl I):P47 doi: I0.I I86/I532-429X-I2-SI-P47

This abstract is available from: http://jcmr-online.com/content/I2/SI/P47

(C) 2010 Xu et al; licensee BioMed Central Ltd.

\section{Introduction}

Whole heart coronary MRA (CMRA) is typically performed with navigator gating because of the extensive data acquisition needed to achieve an isotropic spatial resolution on the order of $1-2 \mathrm{~mm}^{3}$ with full anatomic coverage $(10-16 \mathrm{~cm})$. Previous studies have shown that whole heart CMRA can be performed with either a single [1] or double $[2,3]$ breath-hold (BH) approach using highlyaccelerated parallel imaging. The single breath-hold approach [1] acquires the coil sensitivity data immediately before and after the coronary MRA data within the same cardiac cycle, whereas the double $\mathrm{BH}$ approach acquires coil sensitivity data in a separate $\mathrm{BH}$. The single $\mathrm{BH}$ approach lengthens the time between the T2 and fat suppression pulses to the image acquisition, and the double BH approach may suffer from misregistration. We propose to acquire the coil sensitivity and coronary MRA data in two separate cardiac phases (early systole and mid diastole, respectively) both within a single $\mathrm{BH}$, in order to circumvent the aforementioned problems.

\section{Purpose}

To develop a robust single BH whole heart CMRA scan with isotropic spatial resolution.

\section{Methods}

Experimental studies were performed in 2 healthy volunteers on a $1.5 \mathrm{~T}$ scanner (Siemens;Avanto). The relevant steady state free precession (TrueFisp) pulse sequence parameters are: FOV $360 \times 360 \times 102 \mathrm{~mm}^{3}$, Matrix $224 \times$ $224 \times 64$, slice thickness $1.6 \mathrm{~mm}$, the voxel size is $1.6 \times 1.6$ $\times 1.6 \mathrm{~mm}^{3}$, interpolated to $0.8 \times 0.8 \times 0.8 \mathrm{~mm}^{3}$, acceleration-factor $4 \times 2$ ( 4 in PE and 2 in PA direction), segment 42, TR $3 \mathrm{~ms}$, TE $1.4 \mathrm{~ms}$, partial Fourier in both PE and PA directions (6/8), slice oversampling-rate $12.5 \%, \mathrm{~T} 2$ and fat-suppression preparation pulses were used. Image reconstruction was performed using the 2D GRAPPA technique. Using identical parameters, we compared two cases: 1) double BH approach and 2) single BH approach where the coil sensitivity and image data are acquired at early systole and mid diastole, respectively (Figure 1). To investigate the inter-study variability, we repeated the image acquisitions for both subjects.

\footnotetext{
Results

Figure 2 shows representative images that demonstrate improvement using the single $\mathrm{BH}$ approach. The new single BH approach produced less "pseudo-noise" artifacts than the double $\mathrm{BH}$ approach, owing to improved registration and consistently improved image quality compared with the double $\mathrm{BH}$ approach in both subjects during repeated sessions.
} 


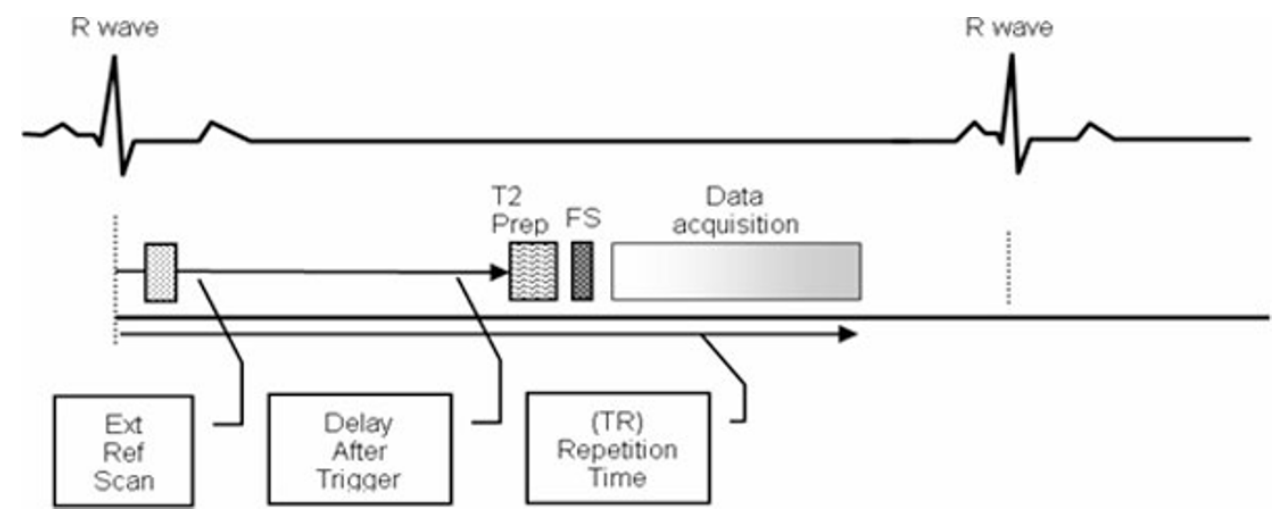

Figure I

Single BH coronary MRA with the coil sensitivity and image data acquired at two different cardiac phases in the same cardiac cycle.

\section{Conclusion}

We demonstrate the feasibility of performing single $\mathrm{BH}$ whole heart CMRA with isotropic spatial resolution. Compared with the double $\mathrm{BH}$ approach, the new single BH approach yielded less "pseudo-noise" artifacts in the GRAPPA reconstruction. This approach has the potential to improve diagnostic accuracy for rapid volumetric CMRA.

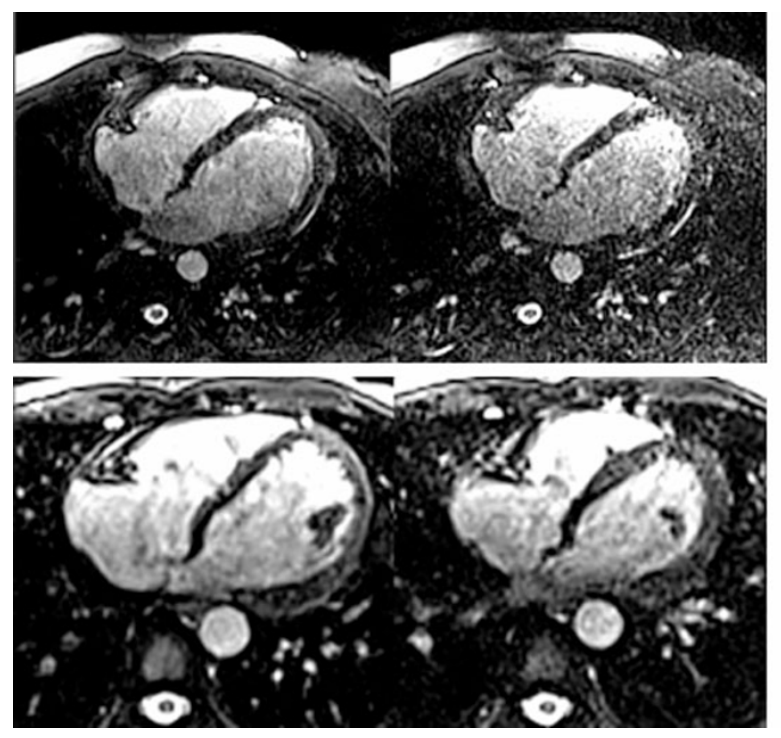

(a)

(b)

Figure 2

Representative images of subject I (top row) and 2 (bottom row): (left) new single BH and (right) double BH.

\section{References}

I. Jaeseaok Park, et al.: High-resolution steady-state free precession coronary magnetic resonance angiography within a breath-hold: parallel imaging with extended cardiac data acquision. Magnetic Resonance in Medicine 2005, B54B: I I 00-I I 06.

2. Thoralf Niendorf, et al.: Toward a single breath-hold wholeheart coverage coronary MRA using highly accelerated parallel imaging with a 32-channel MR system. Magnetic Resonance in Medicine 2006, 56:167-176.

3. Jian Xu, et al.: Feasibility of five-minute comprehensive cardiac MR examination using highly accelerated parallel imaging with a 32-element coil array. ISMRM Honolulu 2009:725.

Publish with BioMed Central and every
scientist can read your work free of charge
"BioMed Central will be the most significant development for
disseminating the results of biomedical research in our lifetime. "
Sir Paul Nurse, Cancer Research UK
Your research papers will be:
- available free of charge to the entire biomedical community
- peer reviewed and published immediately upon acceptance
• cited in PubMed and archived on PubMed Central
- yours - you keep the copyright
hubmit your manuscript here:

